\title{
Patterning Biological Molecules onto Poly(amidoamine) Dendrimer on Gold and Glass
}

\author{
Mi-Young Hong, Dohoon Lee, Hyun C. Yoon, ${ }^{\star}$ and Hak-Sung Kim \\ Deparment of Biological Sciences, Korea Adwanced Institute of Science and Technologv. Daejeon 305-701. Korea \\ ${ }^{\dagger}$ Division of Chemical Engineering and Biotechnologv, Ajou Chiversin, Paldal-Gu, Simon $+42-7+9$. Korea
}

Received Mav 22, 2003

\begin{abstract}
Patterning of biological molecules was attempted on both gold and glass using fourth generation (Gt) poly (amidoamine) (PAMAM) dendrimer as an interfacing layer between solid surfaces and biomolecules. As for the patterning of avidin and anti-biotin antibody on gold. PAMAM dendrimers representing anine functionalities were firstly printed onto the 11-mercaptoundecanoic acid SAM by microcontact printing. followed by biotinylation, and reacted with fluorescence-labeled avidin or anti-biotin antibody. Fluorescence microscopic analysis revealed that the patterns of avidin and anti-biotin antibody were well constructed with the resolution of $<2 \mu \mathrm{m}$. The PAMAM dendrimers were also printed onto aldehyde-activated slide glass and reacted directly with anti-BSA antibodies. which had been oxidized with sodium periodate. As a result, distinct patterns of the anti-BSA antibodies were also obtained with a comparable edge resolution to that of avidin patterns on gold. These results clearly show that PAMAM dendrimers can be adopted as an interfacing laver for the patterning of biological molecules on solid surfaces with micrometer resolution.
\end{abstract}

Key Words : Patterning. Biomolecules. PAMAM dendrimer. Microcontact printing

\section{Introduction}

Patterning biological molecules on solid surfaces is increasingly attracting attention in many areas including biosensors. controlled cell adhesion and arrays of ligands and biomolecules ${ }^{1-6}$ A number of methods have been reported for the formation of patterns on solid surfaces. and various kinds of interfacing layers have been employed in an effort to precisely control the physicochemical properties on solid surfaces. ${ }^{1.5-1010}$ Over the past few years. microcontact printing. known as a soft lithographic teclunique. has been of great interest. because it offers very simple and versatile tool for the patterning of biomolecules or small compounds on solid surfaces..$^{2.11-15}$ In this technique. an elastomer. typically' made of poly'(dimethylsiloxane) (PDMS) is used as a stamp. and printing of molecules onto surfaces generates microscale patterns of the target molecules. Self assembled monolayers (SAMs) on gold and silicon have been widely used as base substrates for patterning ${ }^{1-3.5 .5 .10 .11 .16}$ and recently different kinds of interfacing layers including linear and copolymerized polymers have been developed extending the utility of microcontact printing technique to other applications. ${ }^{14.17-26}$ It is generally accepted that interfacing layers between solid surfaces and biomolecules have a profound effect on spatial control of the surface or the interface property. surface density of the molecules. and chemical and electrochemical surface reactions.

Highly branched dendritic macromolecules (dendrimers) have been recently recognized as a promising candidate for building units of molecularly organized nanostructures and

"Corresponding author. Phone: +82-42-869-2616; Fax: +82-42869-2610; E-mail: hskim76ậkaist.ac.kr interfacing materials. ${ }^{21.22}$ With unique characteristics such as structural homogeneity. integrity. controlled composition. and multiple homogeneous chain ends available for consecutive conjugation reaction. there have been a number of approaches for the construction of dendrimer-based composites. e.g. deposition of dendritic multilayers via $\mathrm{Pt}^{2-}$ complexation. electrostatic interaction. construction of monolayers on reactive SAMs. $2=-26$ Furthermore the surface groups of dendrimers can be chemically functionalized through synthetic manipulations. extending their applications. ${ }^{77-35}$ Recently dendrimers were also used as an ink for the contact printing ${ }^{36}$ and employed as a linker for the construction of DNA microarrays. ${ }^{37.38}$

To date. we have shown that modified surfaces with PAMAM dendrimer having amine groups can be effectively made onto SAMs/gold substrates and functionalized with chemical and biological ligands for the purpose of biosensors. ${ }^{39.47}$ In addition. monolayers of biomolecules can be constructed on the dendrimer layers through simple coupling reactions. ${ }^{39}$ Based on the previous studies and the abovementioned characteristics of dendrimers. it is expected that dendrimers can be employed as an interfacing layer for the patterning of biomolecules on solid surfaces. In this study. as an approach to the patterning of biological molecules on solid surfaces. we firstly printed dendrimers onto both reactive SAMs on gold and aldelyde-activated glasses by microcontact printing. The dendrimers on gold were functionalized with biotin and reacted with avidin or anti-biotin antibody' and the formation of patterns was investigated by fluorescence microscopy. Patterning of anti-bovine serum albumin (BSA) antibody was attempted through direct reaction with the dendrimers on glass and also examined using the same method. Details are reported herein. 


\section{Experimental Section}

Materials. Poly (dimethylsiloxane) (Sylgard 184) and its curing agent are manufactured by Dow Chemical. 1.2.2(Tridecafluoro-1.1.2.2-tetrahydrooctyl)-1-trichlorosilane was purchased from UTC Inc.. Amine-terminated fourth generation (G4) poly(amidoamine) (PAMAM) dendrimer is manufactured by Dendritech. Inc. (Midland. MI) and was purchased from Aldrich. 1-Ethyl-3-(3-dimethyl aminopropyl)carbodimide hydrochloride (EDAC). sodium cyanoborohydride fluorescein isothiocyanate (FITC). and fluorescein isothiocyanate-labeled avidin (Avidin-FITC. $3.0-5.5 \mathrm{~mol}$ FITC/mol protein) were from Sigma and used as received. 11-Mercapto-1-undecanol (MUOH). 11-mercaptoundecanoic acid (MUA) , pentafluorophenol ( $\mathrm{PF}_{5}$ ). sodium cyanoborohydride ( $5 \mathrm{M}$ in $1 \mathrm{~N} \mathrm{NaOH})$, and 2-(2-aminoethoxy)ethanol (AEE) were purchased from Aldrich. Sodium (meta)-periodate and octadecanethiol (ODT) were from Fluka. Biotinyl$\varepsilon$-amidocaproic acid $N$-hyddroxysulfosuccinimidyl ester (sulfo-NHS-biotin), biotiny 1-3,6-dioxaoctanediamine (biotin PEO-LC-amine) and immunopure avidin were purchased from Pierce. Anti-biotin monoclonal antibody (mouse monoclonal $2 \mathrm{~F} 5$. isoty'pe $\operatorname{IgG}_{1 . \kappa}$ ) as well as its fluorescently labeled form (Alexa Fluor 488 conjugate 5.0-6.0 mol labei/ mol protein) was obtained from Molecular Probes. Antibovine serum albumin (anti-BSA) antibody developed in rabbit was from Sigma and used as received. Fluorescent poly'styrene microbead solution (aldehy'de-coated beads. $2 \%$ solids). whose bead diameter was $20 \mathrm{~mm}$. was received from Molecular Probes and used after proper treatment as recommended by manufacturer.

Thin film gold surfaces were prepared by resistive evaporation of $200 \mathrm{~nm}$ of gold (All. 99.999\%) onto titaniumprimed (Ti. $20 \mathrm{~nm})$ silicon wafers $(\mathrm{Si}[100])$. Aldelydeactivated glass slides were purchased from Telechem (Suuny̧vale. CA).

Preparation of stamps for microcontact printing. For the patterning of biomolecules on solid surfaces. poly(dimethỵlsiloxane) (PDMS) stamps having desired features were made and used for the microcontact printing according to the method developed by Whitesides group. "Briefly. micropatterns of $4 \times 4$ arrays with feature sizes of 100 or 50 $\mu \mathrm{m}$ square corrals were designed by AutoCAD software (see Figure 2). Masks of the designed micropatterns were made by photolithography, and the masters. that were used to cast the PDMS stamps. were fabricated by photoresistor spincoating and processing. The depth of the relief was controlled at about $6 \mu \mathrm{m}$. Patterned stamps were made by pouring a 10:1 mixture of Sylgard 184 PDMS prepolymer and curing agent (wt. ratio) over the silane pretreated master surface in a petri dish. and curing at $70^{\circ} \mathrm{C}$ overnight. The silanization reaction was performed in a desiccator under reduced pressure by placing the master with a few drops of 1.2.2-(Tridecafluoro-1.1.2.2-tetrahy'drooctyl)-1-trichlorosilane for $20 \mathrm{~min}$. After overnight curing the hardened PDMS stamp was peeled off from the master and washed with ethanol and ddw (doubly distilled water). The master could be repeatedly used without severe contamination when the silanization process was performed satisfactorily. Thickness of the resulting stamps was controlled at about 5-7 mm for the ease of inking and microcontact printing.

Printing amine-terminating dendrimers onto gold and glass. For the printing on gold. MUA SAM was prepared on gold and activated as described elsewhere ${ }^{25+1}$ Methanolic dendrimer solution $(0.7 \mathrm{mM})$ was used as an ink material for the microcontact printing process. A newly prepared PDMS stamp was inked by scnubbing the surface with a cotton swab soaked with the dendrimer solution and subjected to an argon flow for solvent evaporation. After drying. the inked stamp was brought into conformal contact with the $\mathrm{PF}_{5-}$ activated and dried SAM/Au surface. The duration of the contact between the stamp and the activated SAM was adjusted to $5 \mathrm{~min}$ for the effective transfer and reaction of dendrimer molecules on the solid surface. The stamp was peeled off with care for not fouling the printed patterns. After peeling off the PDMS stamp. the surfaces were rinsed with methanol. dried with a stream of argon. and immersed in a bicarbonate buffer $(0.1 \mathrm{M}, \mathrm{pH} 9.5)$ for 20 min to hydrolyze the remaining fluorophenyl esters.

For the printing of dendrimers on glass. aldehyde-activated slide glasses were used and subjected to microcontact printing without preactivation. The resulting glasses were incubated in AEE ethanol solution (l M) for $2 \mathrm{~h}$ to block the aldelyde functionalities remaining in non-patterned regions. The Schiff bases formed by amine-aldelyde coupling were reduced by adding $10 \mu \mathrm{L}$ of $5 \mathrm{M}$ sodium cyanoborolydride in $1 \mathrm{~N} \mathrm{NaOH}$ per $1 \mathrm{~mL}$ reaction volume and the reaction proceeded for $2 \mathrm{~h}$. followed by thorough washing with ethanol and ddw. To this step. the patterning of dendrimers was completed for further bio-functionalization.

Mixed-SAM supported pattem preparation. For printing. ODT was used as the ink. because this compound showed a sufficient hydrophobicity for wetting the untreated PDMS stamp (not treated with oxygen plasma), and presented a good pattern of ligands. After microcontact printing with ODT, which is essentially the same procedure to that with dendrimers. the resulting surfaces were treated with a mixed solution of thiols containing reactive functionality: A mixture of MUOH and MUA $(10: 1$, w/w) was prepared and used to back-fill the remaining surface regions after ODT printing because this composition was found to show the best result when avidin-specific monolay'ers are made on the biotinylated mixed-SAM. ${ }^{\text {t2 }}$ After filling reaction with a thiol-mixture and washing. biotinylation reaction was proceeded with biotin PEO-LC-amine wia $\mathrm{PF}_{5}$ activation of the surface carboxylate groups from adsorbed MUA.

Association of fluorescence-labeled and aldehyde-functionalized microbeads onto the printed surface. To confirm the printing of amine-terminated dendrimers on either gold or glass. pattern visualization wia fluorescence microscopy was conducted by reacting with aldehyde-coated fluorescent microbeads (diameter $=20 \mathrm{~nm}$ ). Bead solution was freshly prepared before each experiment by diluting it with $\mathrm{ddw}$ to $0.2 \%$ solid content. Aggregates were removed by centrifug- 
ing the solution for $3 \mathrm{~min}$ under $10.000 \mathrm{~g}$ and decanting precipitate following manufacturer's recommendation. The resulting fluorescent microbead solution was applied to gold or glass surfaces representing printed dendrimers. After one hour of amine-aldehyde surface reaction. the resulting surface was dipped into sodium cyanoborohydride solution $(5 \mathrm{mM}$ in ddw. $20 \mathrm{~min}$ ) for the reduction of Schiff bases formed. The resulting surfaces were subjected to thorough washing steps with $\mathrm{ddw}$ and PBST $(\mathrm{pH} 7.4,10 \mathrm{mM}$ phosphate. $2.7 \mathrm{mM} \mathrm{KCl} .138 \mathrm{mM} \mathrm{NaCl}$ and $0.05 \%(\mathrm{v} / \mathrm{v})$ Tween 20), followed by visualization experiment using fluorescence microscopy:

Patterning avidin and anti-biotin antibody. To form the patterns of avidin and anti-biotin antibody on either gold or glass the printed dendrimers were firstly biotinylated using sulfo-NHS-biotin as described in our previous work. ${ }^{+1}$ For pattenning of avidin. FITC-avidin solution $(1 \mu \mathrm{M}$. based on the avidin concentration) in PBST was applied to the patterned/biotinylated surface for $20 \mathrm{~min}$ in the dark after rinsing with PBST. The surfaces were then thoroughly washed with PBST and ddw. Subsequently, the fluorescent patterns of the surface were investigated using fluorescence microscopy. Similar procedure was employed for the patteming of anti-biotin-antibody. and Alexa488-labeled antibody was used. Antibody solution in PBST $0.02 \mathrm{mg}$ antibody $/ \mathrm{mL}$ ) was applied for $30 \mathrm{~min}$ in the dark. and the resulting surface was thoroughly rinsed with PBST and ddw before pattern analysis.

Patterning anti-BSA antibody. Anti-BSA antibody was firstly labeled with FITC as described elsewhere. ${ }^{43}$ and FITC-labeled antibodies were used immediately in the following experiments. To directly couple the antibody with the amine-terminated dendrimer molecules. the carbohydrate moieties of anti-BSA antibody were oxidized with sodium periodate ${ }^{34.44}$ Briefly sodium periodate $(20 \mathrm{mM})$ was added to anti-BSA-FITC antibody solution $(0.4 \mathrm{mg} / \mathrm{mL})$ in sodium acetate buffer $(150 \mathrm{mM} . \mathrm{pH} 5.2)$. The reaction proceeded in the dark for $1 \mathrm{~h}$ at room temperature with mild stirring and was quenched by addition of $10 \mu \mathrm{L}$ ethylene glycol for $30 \mathrm{~min}$. The modified antibody molecules were purified in Centriprep ${ }^{8}$ (Millipore. MA) following manufacturer's instruction through 8-time buffer changes. and finally: the solution was concentrated to $1 \mathrm{mg}$ antibody $/ \mathrm{ml}$. For the patterning of FITC-labeled anti-BSA antibody on either gold or glass. the dendrimer-printed surfaces were rinsed several times with PBST buffer before binding reaction. The surface was immersed in the anti-BSA-FITC (with exposed aldehyde functionality on the carbohydrate moiety) solution (0.02 $\mathrm{mg}$ antibody $/ \mathrm{mL}$ in PBST) for $20 \mathrm{~min}$ in the dark. Then the surface was rinsed thoroughly with PBST and ddw before observation with fluorescence microscope.

Instrumentation. Olympus BX40 laboratory microscope with URA fluorescence attachment was used for the fluorescence microscopy. Images were collected by a cooled Photometrics SenSys CCD camera (Roper Scientific GmbH. Germany) and saved on an Apple PowerMac through Photometrics SCSI A2S Box (Roper Scientific GmbH. Germany).

\section{Results and Discussion}

Printing PAMAM dendrimer on gold. For the pattening of biomolecules on gold. PAMAM dendrimers representing anine functionalities were firstly printed onto SAMs of 11mercaptoundecanoic acid by microcontact printing. The procedure is schematically shown in Figure 1. The reaction between amine groups from the dendrimer chain-ends and the surface ester groups from the activated-SAMs was confirmed through an electrochemical method as reported in our previous studies. ${ }^{39-41}$ SPR analy'sis revealed that the covalent coupling yield of dendrimers to the ester-activated SAMs by microcontact printing is about $80 \%$ of that by simple inumersion reaction (data not shown).

It might be noteworthy that in addition to the anticipated merits from the nature of dendrimers. there were some unexpected advantages in using dendrimer as a printing ink material. For the successful constnuction of micropatterns on surfaces by microcontact printing. enough amount of ink material should be delivered to the surfaces. and the efficient wetting of stamps with ink is a crucial factor. In case of PDMS stamp. oxygen plasma treatment is usually conducted to render the surface more hydrophilic for the purpose. ${ }^{1]}$ However, when PAMAM dendrimer was used as the ink material. stamp surface could be readily wetted by a methanolic solution of dendrimer. and the oxygen plasma treatment was not necessary.

To examine the printing of dendrimers on reactive SAMs

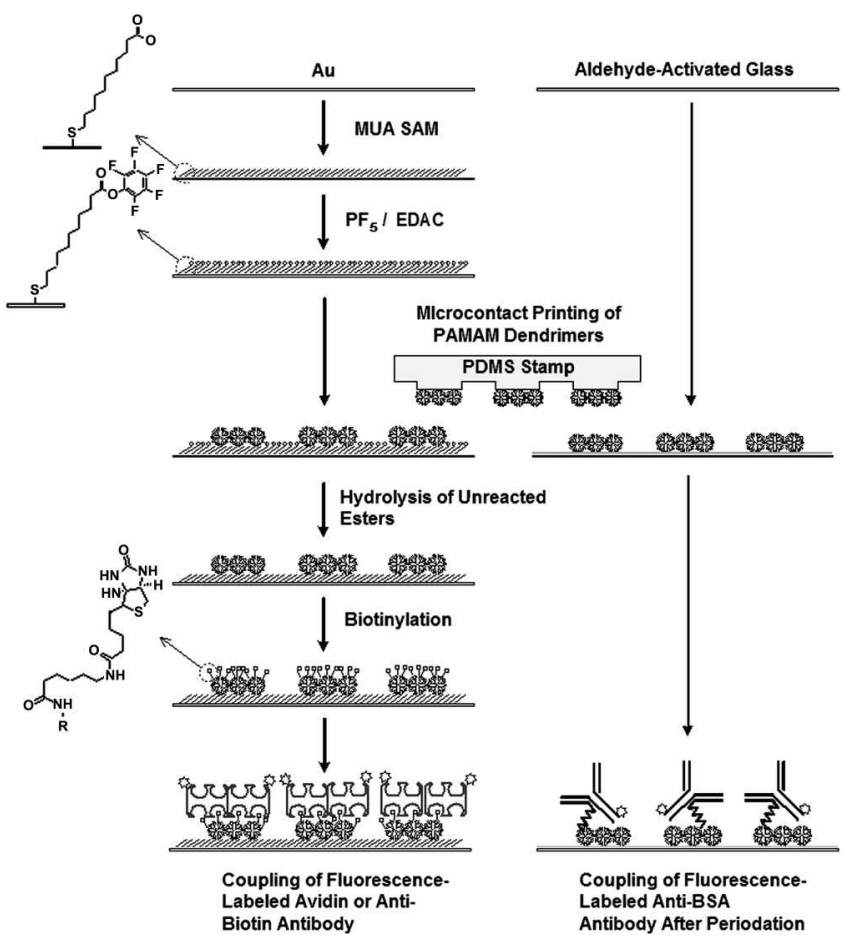

Figure 1. Schematic representation of the microcontact printing and patterning of biomolecules on gold and glass. The dimensions of the components are not drawn to scale for simplicity (MUA: 11 mercaptoundecanoic acid, PFs: pentafluorophenol, EDAC: 1-ethyl3-(3-dimethyl aminopropyl)carbodimide hydrochloride, PAMAM: poly(amidoamine), PDMS: poly (dimethylsiloxane) 
(A)
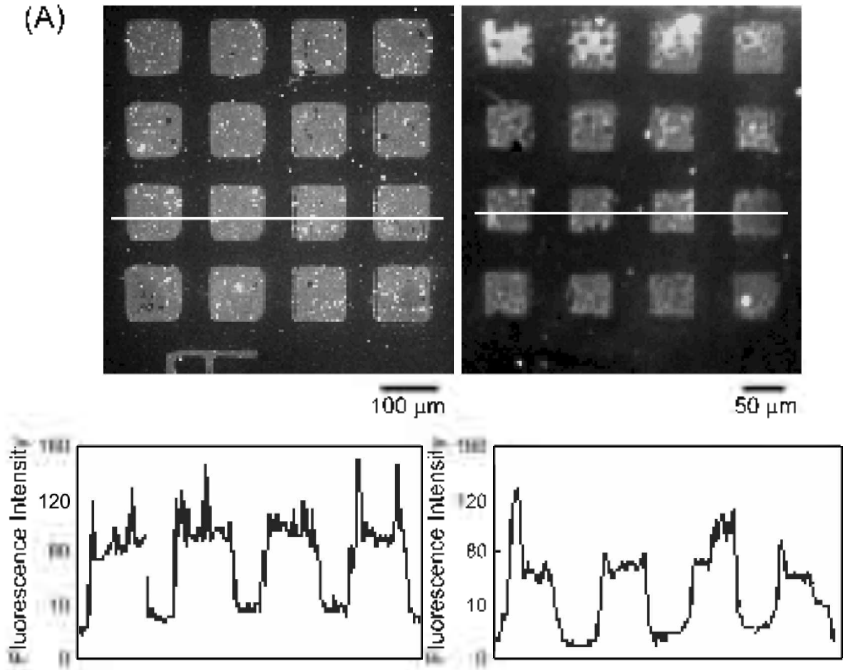

(B)
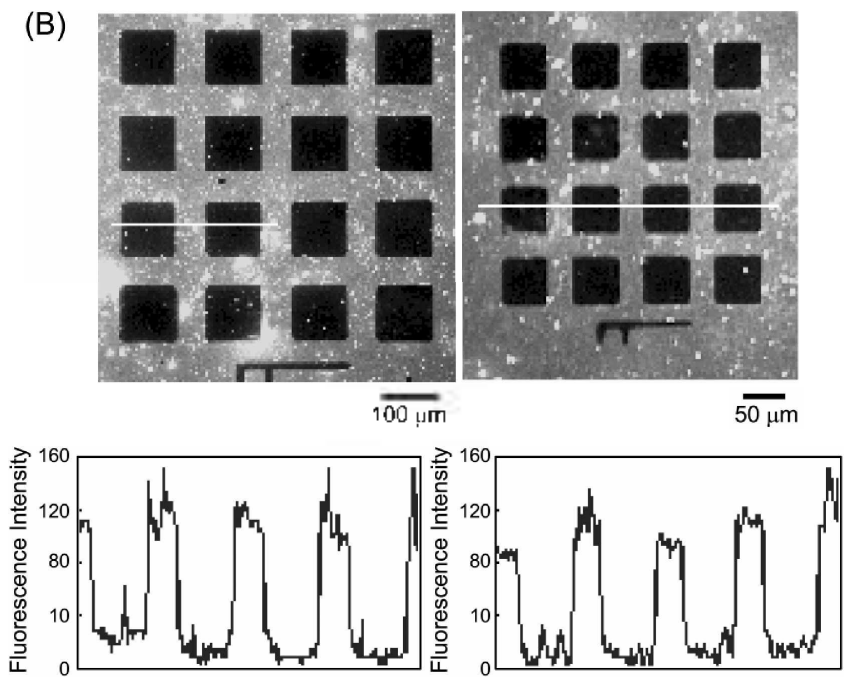

Figure 2. Illoresenese images of the dendrimer-printed gold surface incubated with fluorescent microbeads. Positive (A) and negative (B) patlerns with the leature sizes of $100 \mu \mathrm{m}$ and $50 \mu \mathrm{m}$ arc shown with scale bars. Also shown are lluorescence profiles across the white lines drawn on images.

on gold, the printed surface was incubated with fluorescent microbeads having anine-reactive aldehyde groups at the beads surfaces. The microbead has tluorescent dyes in its cavity and surface aldehyde groups, which can react with the amine - terminating dendrimers for coupling reaction. Figure 2 shows the images of the patterned surfaces. The $4 \times 4$ arrays of rectangles with side lengths of $100 \mu \mathrm{m}$ and $50 \mu \mathrm{m}$ were successfully constructed and could be clearly seen. Stamps of positive and negative patterns were made from the same photomask, and each stamp was used to print dendrimers on the activated-SAMs/gold substrate simultaneously. The estimated resolution of this patterning technique is $<2$ $\mu \mathrm{m}$. which is similar to that reported elsewhere. "This level of resolution is sufticient for most biotechnological applications. ${ }^{2}$ Recently, sub-100 nm scale patterning of dendrimers on silicon through contact printing was reported. ${ }^{36}$

Io form the patterns of biomolecules on the dendrinerprinted gold. the surface was functionalized with biotin
(A)
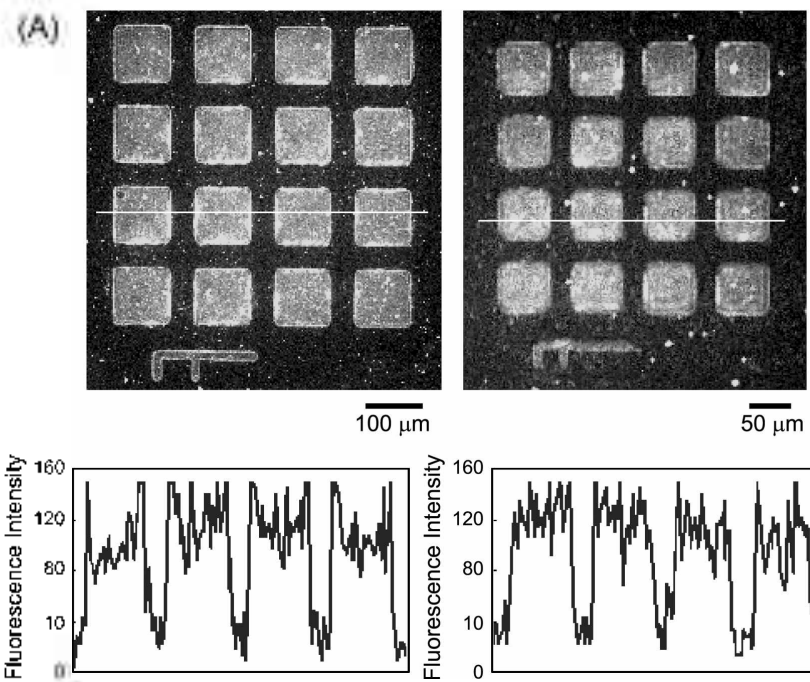

(B)
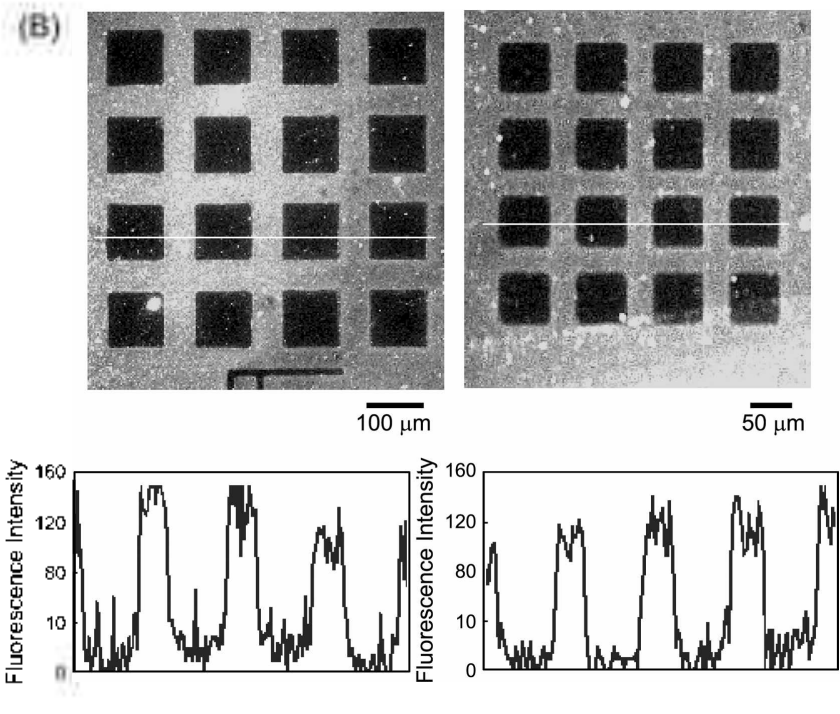

(C)
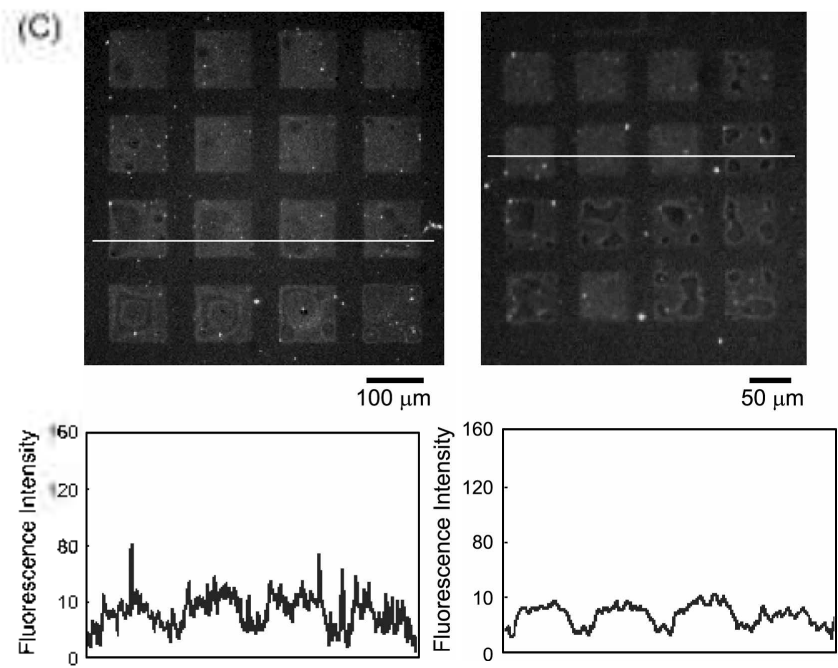

Figure 3. Fluorescence images of the avidin pattens construcled on the dendrimer-printed gold surface (A and $B$ ) and on the mixedSAM (C) which had been moditied with biotin ligands. FITClabeled avidin was used. Positive ( $A$ and $C$ ) and negative (B) patlerns with the feature sizes of $100 \mu \mathrm{m}$ and $50 \mu \mathrm{m}$ are shown with seale bars and fluorescence profiles across the white lines drawn on images. 
groups. and FITC-labeled avidin was applied to the biotinylated surface as described in the experimental section. Figure 3 shows the image of the patterned surface under fluorescence microscopy. and distinctive patterns of avidin were observed. The edge resolution of the patterns was estimated to be about $2 \mu \mathrm{m}$. As a control set for comparison with the dendrimer-assisted pattems. a mixed-SAM supported pattems were also prepared and imaged with FITC-labeled avidin. For the mixed-SAM supported patterns (C) the patterns were also readily discernible. but. the fluorescence intensity' was weaker compared to that of the dendrimer-assisted patterns. The dendrimer-assisted patterns were estimated to result in about 3-3.5 times stronger intensity (that is. the amount of immobilization) of biospecific interaction compared to the mixed-SAM assisted pattems.

As an extension of the approach to other biomolecules. FITC-labeled anti-biotin antibody molecules were applied to the dendrimer-printed surface using identical procedure as shown in Figure 1. As a result. fluorescence microscopy image of the pattemed antibody was clearly observed (Figure 4(A)). A high contrast between pattemed and non-patterned regions. similar to that of the surfaces pattemed with FITClabeled avidin molecules as in Figure 3, was obtained. However. the edge resolution and overall signal intensity of the antibody patterns were not as good as that with avidin molecules. This result might be attributed to the low association constant of the biotin/anti-biotin pair compared to that of the avidin/biotin couple. ${ }^{45}$

Based on the above results. it is expected that dendrimer molecules can be printed on reactive-SAMs on gold in a relatively simple and straightforward way thereby acting as a platform for patteming of biomolecules. This seems to come from the unique characteristics of highly branched dendrimer molecules with high-density functional groups for further modification or immobilization of biomolecules. For example the fourth generation (G4) PAMAM dendrimer used in this work has 64 amine functional groups at its surface and amine is a very versatile functionality in bioconjugation chemistry. Other amine-polymers. such as poly'(L-lysine) and poly'aryl)amine were also tested but dendrimer was found to result in more fasorable platform for further biofunctionalization.

Patterning of antibody on glass. To examine the usefulness of dendrimer as an interfacing layer for the patterning of biomolecules on glass. we employed aldehyde-activated glass slides. In this case. the dendrimers can be directly printed and coupled with aldehyde functional groups on the glass as depicted in Figure 1. As a typical antibody. antiBSA antibody was used after labeling with FITC. The carbohydrate moieties of the anti-BSA antibody were oxidized with periodate ${ }^{44}$ and reacted with the dendrimerprinted glass surfaces. Non-pattenned region was filled with hydroxyl-terminating molecule. 2-(2-aminoethoxy')-ethanol (AEE), by considering its characteristic resistance against non-specific protein binding. ${ }^{47}$ Figure 4 (B) shows the result of fluorescence microscopic analysis from the surface. and a distinct pattern was observed. However. the overall fluores-
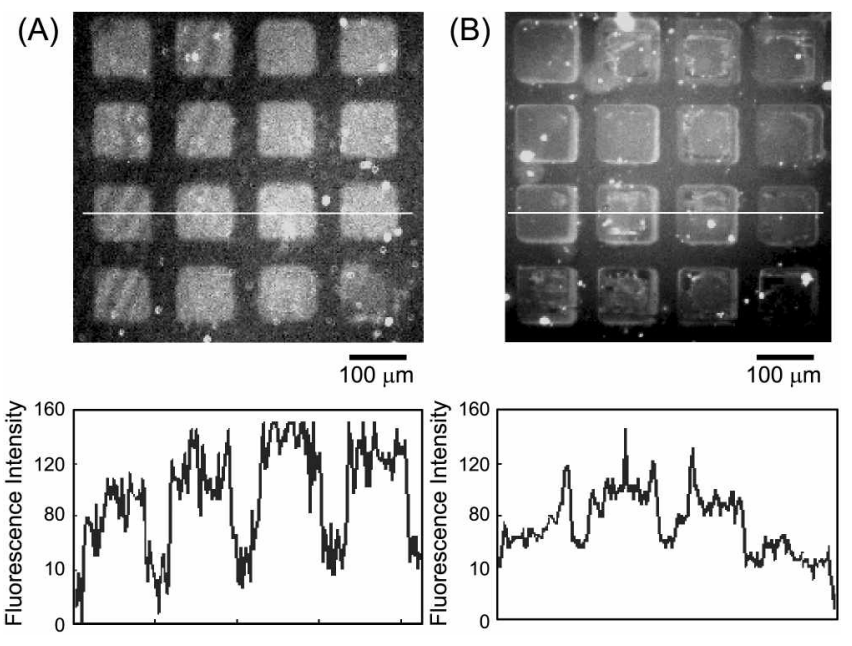

Figure 4. (A) Fluorescence image of the anti-biotin antibody patten fabricated on the dendrimer-printed gold surface which had been biotinylated. Alexa Fluor 488 - labeled antibody was used Also shown is the tluorescence protile across the white line drawn on the image. $(B)$ Fluorescence image of the anti-BSA antibody pattem constructed on the dendrimer-printed glass. FITC-labeled anti-BSA antibody was oxidized with sodilum periodate and directly coupled with the dendrimer-printed glass. A positive pattem with $100 \mu \mathrm{m}$ rectangles is shown with a scale bar and the fluorescence protile across the white line drawn on the image.

cence intensity and especially the contrast between patterned and non-patterned regions were relatively low compared to those of the avidin/biotin couple. This seens to be due to low fluorescent labeling yield of the protocol employed (0.3$1.0 \mathrm{~F} / \mathrm{P}$ in general ${ }^{+3}$ compared to $3.0-5.5$ for avidin and $5.0-$ 6.0 for anti-biotin antibody) and/or to the sticky nature of the antibody, which promotes non-specific binding. In addition. self-conjugation between modified antibodies might lead to low coupling yield of antibody molecules onto the printed dendrimers.

\section{Conclusions}

We have described a method for the patterning of biological molecules such as protein and antibody on gold and glass surfaces by using PAMAM dendrimers as an interfacing lay'er. Printing of the PAMAM dendrimers was readily carried out on the reactive SAMs on gold as well as on the aldehyde-coated glass by the microcontact printing method. Visualization of the dendrimer-printed surface indicated that the PAMAM dendrimers could be efficiently' transferred to the gold and glass surfaces even without pretreatment of the PDMS stamp. forming the uniform layers on the surfaces. Fluorescence microscopic analyses also revealed that the pattens of biological molecules such as protein and antibody were well constructed on the dendrimer-printed surfaces with micrometer resolution. Based on these observations. it is plausible that PAMAM dendrimers can be effectively adopted as an interfacing layer for the patterning of biomolecules on solid surfaces in a relatively simple way. 
Acknowledgement. This research was supported in part by the BK21 Program of the Ministry of Education. the National Research Laboratory Program, and IMT-2000 Program of the Ministry of Science and Technology: Korea.

\section{References}

1. Blawas. A. S.: Reichert. W. M. Biomaterials 1998. 19. 595.

2. Kane, R. S: Takavama, S.: Ostuni. E.: Ingber. D. E.: Whitesides. G. M. Biomaterials 1999, 20.2363.

3. Mrksich. M.: Whitesides. G. M. Trends Biotechnol. 1995. 13. 228.

4. Chen. C. S.: Mrksich. M.: Huang. S.: Whitesides, G. M.: Ingber. D. E. Biotechnol. Prog. 1998, H4. 356.

5. Yousaf. M. N.: Holseman. B. T.: Mrksich. M. Proc. Matl Acad. Sci. LS.A $2001,98,5992$

6. Quan. D.: Kim. Y: Yoon. K. B.: Shin. W. Buhl. Korean Chem. Soc. 2002. 23. 385.

7. Mooney. J. F.: Hunt. A. J.: McIntosh. J. R.: Liberko. C. A.: Walba. D. M.: Rogers. C. T. Proc. Natl Acad Sci LS.A 1996. 93, 12287.

8. Vaidva. R.: Tender L. M.: Bradley. G.: OBrien II, M. I.: Cone, M: López, G. P. Biotechol. Prog 1998. 14, 371.

9. Brooks. S. A.: Ambrose. W. P.: Kulır. W. G. Anal. Chem. 1999. 71. 2558.

10. Yang. Z: Frey. W.: Oliver. T.: Chilkoti. A. Langmir 2000. I6. 1751 .

11. Lahiri, I.: Ostuni, E.; Whitesides, G. M. Langmuir 1999, I5. 2055 .

12. Wilner. I.: Schlittner. A.: Doron. A.: Joselevich. E. Lamgmin 1999. 15.2766

13. Kung. L. A.: Kam. L.: Hovis. J. S.: Boxer. S. G. Langmuir 2000. 16.6773 .

14. Inglis. W. Sanders. H. W.: Willians. P. M: Davies, M. C: Roberts. C. J.: Tendler: S. I. B. Lamgmmir 2001. 17. 7402.

15. Lee. K.-B.: Kim. Y.: Choi. I. S. Buhl. Korean Chem. Soc, 2003. 24. 161 .

16. Kumar. A.: Abbott. N. L.: Kim. E.: Biebuyck. H. A.: Whitesides. G. M. Acc. Chent Res. 1995, 28.219

17. Frey. B. L.: Corn. R. M. Anal Chem. 1996. 68, 3187.

18. Franchina. J. G.: Lackowski, W. M.: Dermody. D. L.: Crooks. R. M: Bergbreiter. D. E. Anal Chem. 1999.71.3133.

19. Yan. L.: Huck. W. T. S.: Zhao. X.-M: Whitesides. G. M. Langmit 1999. 15.1208

20. Ruiz-Tavlor L. A: Martin, T. L: Zaugg. F. G.: Witte. K: Indermulhle. P.: Nock, S: Wagner. P. Proc Natl Acad Sci. LS.A $2001,98,852$.

21. Fischer. M:: Vögtle. F. Angew: Chem. Int Ed 1999. 38. 884.
22. Tully D. C.: Frechet, J. M. J. Chent Commm. 2001. 1229.

23. Regen, S. L.; Watanabe. S. J. Am. Chem. Soc. 1994. 116, 8855.

24. Tsukruk. V. V: Rinderspacher. F.: Bliznyuk. V. Langmair 1997. 13. 2171 .

25. Wells. M.: Crooks. R. M. J.Ant Chent Soc. 1996. 118. 3988.

26. Crooks. R.: Ricco. A. J. Acc. Chent Res 1998, 31. 219

27. Cuadrado, I.: Moran, M. Casado, C. M; Alonso. B; Lobete. F.: Garcia. B: Ibisate. M.: Losada, J. Orgonometallics 1996, 15 5278 .

28. Takada. K.: Diáz. D. T.: Abruña. H. D.: Cuadrado. I.: Casado. C.: Alonso. B.: Morán. M.: Losada. J. J. Am. Chem. Soc. 1997. 19 10763 .

29. Tabakovic, I; Miller. L. L.: Duan, R. G.; Tully, D. C.; Tomalia. D. A. Chem Hater 1997.9.736.

30. Valério. C.: Fillaut. J.-L.: Ruiz. J.: Guittard. ..: Blais. J.C.: Astruc. D. J. Am. Chem Soc. 1997. 119. 2588.

31. Wilbur. D. S.: Pathare. P. M.: Hamlin. D. K: Buhler. K. R: Vessella, R. L. Bioconjugate Chem. 1998. 9.813.

32. Singh. P. Bioconimgate Chent $1998,9,54$.

33. Anzai. J.-I.: Kobayashi, Y.; Nakamura. N.: Nishimura, M.: Hoshi, T. Langmit 1999. 15. 221.

34. Yoon. H. C.: Hong. M.-Y: Kiml. H.-S. Anal Chem. 2000. 72. 4420 .

35. Yoon, H. C.; Kim. H.S. Anal. Chem. 2000, 72.922

36. Li. H.; Kang. D.-J.: Blamire. M. G.: Huck. W. T. S. Nano Lett. 2002. 2, 347.

37. Benters. R.: Niemever. C. M.: Wöhrle. D. Chem. Biol. Chem. 2001. 2. 686 .

38. Benters. R.: Niemeyer. C. M.: Drutschmann. D.: Blohmn. D.: Wohrle. D. Nucleic Acids Res 2002. 30. el0

39. Yoon. H. C.: Hong. M.-Y; Kim, H.-S. Anal Biochem. 2000, 282. 121 .

40. Yoon1. H. C.: Hong. M.-Y.: Kim. H.-S. Langmair 2001. 17. 1234.

41. Yoon. H. C.: Lee. D.: Kim. H.-S. Anal Chim Acta 2002. 456. 209.

42. Jung. L. S.: Nelson. K. E.: Stavton, P. S.: Campbell, C. T. Langnuir 2000. 16.9421

43. Harlow E; Lane D. Lsing Anthodies: A Laboraton Manat; Cold Spring Harbor Laboratory Press: Cold Spring Harbor. New York. U. S. A.. 1999: p 85.

44. Hermanson. G. T. Bioconingate Techmiques: Academic Press: San Diego, California U. S. A., 1996: p 475 .

45. Lynch. N. J. Kilpatrick. P. K; Carbonell, R. G. Biotechnol. Bioeng. 1996, 50. 169.

46. Hong. M.-Y.: Yoon. H. C.: Kim. H.-S. Langmin 2003. 19. 416.

47. Knoll. W.: Liley. M.: Piscevic. D.: Spinke. T.: Tarlow. M. J. Ad. Biophs: 1997. 3+.231. 